\title{
Sclerotization in Relation to Plasmodial Senescence in the Acellular Slime Mould Didymium iridis
}

\author{
By TIMOTHY LOTT*† AND JIM CLARK \\ T. H. Morgan School of Biological Science, University of Kentucky, Lexington, \\ Kentucky 40506, U.S.A.
}

(Received 13 August 1981; revised 19 October 1981)

\begin{abstract}
When subjected to dehydration, Didymium iridis plasmodia differentiate into sclerotia which can be maintained for long periods and exhibit no discernible $\mathrm{O}_{2}$ uptake. While actively growing plasmodia display the phenomenon of senescence, this ageing is delayed in sclerotia by the duration of the sclerotized state. Upon rehydration, plasmodia live the remainder of their characteristic life spans. Hence, the timing mechanism for senescence is conserved during this reversible differentiation pathway.
\end{abstract}

\section{INTRODUCTION}

In sexual isolates of the myxomycete Didymium iridis, plasmodia are formed by the fusion of two haploid myxamoebae (Dee, 1975), followed by mitotic divisions to create a large syncytium. Once formed, a plasmodium can be environmentally induced to follow one of several differentiation pathways. Sporulation (meiosis) will normally occur after a period of starvation, thus completing the life cycle, but under adverse conditions such as low temperature or dehydration (Jump, 1954) a plasmodium will develop into a more resistant sclerotial stage. Unlike sporulation, sclerotization is a reversible process and redifferentiation will occur rapidly in a moist, temperate environment.

We have previously reported that $D$. iridis plasmodia derived from myxamoebae of the Hon 1 sexual isolate have finite life spans when grown as solid surface cultures on rolled oats (Lott \& Clark, 1980). After several months there is a decline in growth, accompanied by cell necrosis and eventual death. The factors determining longevity are associated with genotype, and both short- and long-lived individuals can be created by the mating of appropriate haploid gametes (Lott \& Clark, 1980). Plasmodial ageing (senescence) has also been observed in isolates of the closely related species Physarum polycephalum (McCullough et al., 1973; Poulter, 1969; Daniel \& Baldwin, 1964).

The molecular basis for the timing of necrosis is not known, although there is evidence suggesting that the process does not involve a simple accumulation or degradation of one or more cellular components. For example, when age-hybrid plasmodia are formed by the fusion of young and old individuals in equal amounts, the hybrid tends to acquire the remaining life span of the older segment (Clark \& Hakim, 1980). In order to further characterize the nature of this timing mechanism, it was of interest to determine the relationship of sclerotization to the onset of senescence.

\section{METHODS}

The numeric nomenclature for both haploid myxamoebae and diploid plasmodia follows that of Collins (1961). The initial formations of short- and long-lived plasmodia (genotypes 10.76 and 2.7, respectively) were performed in $60 \times 20 \mathrm{~mm}$ Petri dishes containing half-strength corn meal agar as previously described (Collins, 1961).

† Present address: Biology Department, Guyot Hall, Princeton University, Princeton, New Jersey 08540, U.S.A. 
Following formation, cultures were transferred on a $7 \mathrm{~d}$ schedule and maintained on $2 \%(w / v)$ non-nutrient agar and rolled oats (Lott \& Clark, 1980). Separately maintained cultures, established from the subdivision of a single plasmodium, are termed sub-division populations. Plasmodial life spans were calculated from the day of the cross to the day of spheroid formation.

For $Q_{\mathrm{O}}$, determinations, plasmodia were sectioned $4 \mathrm{~d}$ after transfer and approximately $4 \mathrm{~cm}^{2}$ segments were placed on non-nutrient agar plates. After $12 \mathrm{~h}$ migration, $17 \mathrm{~mm}$ diameter circular sections were cut in the plasmodial front region. Four segments from each plasmodium were transferred to a double side-armed manometer flask containing $10 \%(\mathrm{w} / \mathrm{v}) \mathrm{KOH}$, and allowed to equilibrate at $22{ }^{\circ} \mathrm{C}$ for several hours on a Gilson respirometer. Readings were taken every $30 \mathrm{~min}$ for $3 \mathrm{~h}$. The plasmodial segments were then removed and transferred to $10 \mathrm{ml} 50 \mathrm{~mm}$-Tris/ $\mathrm{HCl}$ buffer, $\mathrm{pH} 8 \cdot 0$, at $4{ }^{\circ} \mathrm{C}$, and homogenized in a Wheaton glass tissue grinder with the pestle attached to a variable speed motor run at approximately $700 \mathrm{rev}$. $\mathrm{min}^{-1}$. The resulting suspension was centrifuged at $3000 \mathrm{~g}$ for $10 \mathrm{~min}$, and the supernatant was used for protein determinations by the Lowry method. The rate of $\mathrm{O}_{2}$ consumption is expressed as $\mu 1 \mathrm{~min}^{-1}$ (mg protein) ${ }^{-1}$.

Sclerotial $\mathrm{O}_{2}$ uptake was measured at the same time as that of the plasmodial cultures, using a similar procedure. Three cultures from $60 \times 15 \mathrm{~mm}$ dishes were placed in each flask. After removal, the cultures were rehydrated and grown for several days to confirm their viability.

Plasmodial cultures were sclerotized by dehydration using a procedure modified from that of Jump (1954). Normally maintained $4 \mathrm{~d}$ cultures were sectioned and approximately $1 \mathrm{~cm}^{2}$ segments transferred to $60 \times 15 \mathrm{~mm}$ Petri dishes containing $3.0 \mathrm{ml} 2 \%(\mathrm{w} / \mathrm{v})$ non-nutrient agar and $100 \mathrm{mg}$ oats. After $24 \mathrm{~h}$ the original agar blocks were removed and the plates were uncovered, thus exposing the cultures to air at room temperature $\left(22^{\circ} \mathrm{C}\right)$. In general, a $90 \%$ loss of moisture (net weight of agar and plasmodia) occurred within $3 \mathrm{~d}$, resulting in the formation of a brittle agar film containing the sclerotium. At this time the lids were replaced and the cultures were stored at $22{ }^{\circ} \mathrm{C}$. An individual plate will subsequently be referred to as a sclerotial subdivision.

For revival, the entire contents of a single plate were transferred to a $100 \times 15 \mathrm{~mm}$ dish containing $2 \%$ non-nutrient agar. Sterile water $(3.0 \mathrm{ml})$ was then added to the culture. After 30 min the excess water was removed and the plates were streak-inoculated with Escherichia coli. Cytoplasmic streaming was generally observed within $24 \mathrm{~h}$ of the addition of water. When the plasmodium had migrated off the original agar block, it was sectioned. A segment was then transferred to a fresh plate with oats and maintained as normal. The period of sclerotial existence was determined from the beginning of dehydration to the addition of water to the culture.

\section{RESULTS}

Didymium iridis sclerotia were characterized by the formation of wall-like structures dividing the plasmodium into small, irregular units. When created by evaporative dehydration, there was no visible evidence of metabolic activity and no discernible $\mathrm{O}_{2}$ uptake (Table 1). Plasmodia of both short- (10.76) and long-lived (2.7) genotypes had similar rates of $\mathrm{O}_{2}$ consumption, despite the observed twofold difference in average life span between the two strains (Lott \& Clark, 1980).

Plasmodia of genotypes 10.76 and 2.7 readily formed sclerotia when dehydrated, completing the process within $48 \mathrm{~h}$. Revival occurred at approximately the same rate and was marked by the degeneration of cross-walls and the reoccurrence of cytoplasmic streaming. Sclerotium viability averaged approximately $85 \%$ for both strains, with no apparent correlation between the age of the initial culture and the viability of the sclerotium.

To determine if either the process of sclerotium formation or the length of time as a sclerotium had any influence on the timing of senescence, segments of short- and long-lived plasmodia were periodically sclerotized and then revived upon the death of the initial culture. Following revival, cultures were maintained normally and scored daily for deaths. It was observed that life spans, as determined by the number of days as a plasmodium, were highly similar for the initial culture and sclerotial subdivisions. Sclerotia of genotype 2.7 that were formed at a given time prior to the death of the initial culture generally lived that period of time after being revived (Fig. 1): a regression analysis around the theoretical one-to-one relationship was significant $(r=0.853)$. This relationship was also seen for sclerotial subdivisions of genotype 10.76 , as well as in a similar set of experiments where cultures were maintained for a constant period of time before being revived. Sclerotia have been revived successfully after 1 year, and have still maintained a total vegetative life span similar to that of the initial culture. Thus, the length of time as a sclerotium chronologically postponed the 
Table 1. Rates of oxygen consumption by short-and long-lived plasmodia and sclerotia

$\begin{array}{ccc}\text { Genotype } & \begin{array}{c}\text { Relative age } \\ \text { when assayed* }\end{array} & \begin{array}{c}Q_{\mathrm{O}_{2}}^{\dagger} \\ {\left[\mu \mathrm{O} \mathrm{O}_{2}(30 \mathrm{~min})^{-1}\right.} \\ \left(\mathrm{mg} \mathrm{protein}^{-1}\right]\end{array} \\ 2.7 \text { (plasmodium) } & \text { Young } & 129 \pm 31 \\ & \text { Old } & 182 \pm 67 \\ 10.76 \text { (plasmodium) } & \text { Young } & 120 \pm 20 \\ & \text { Old } & 126 \pm 21 \\ 2.7 \text { (sclerotium) } & \text { Young } & 0.0\end{array}$

\footnotetext{
* Young denotes age less than or equal to $25 \%$ of the characteristic genotypic life span. Old denotes age greater than or equal to $75 \%$ of the characteristic life span.

+ Mean value \pm standard deviation $(n=10)$.
}

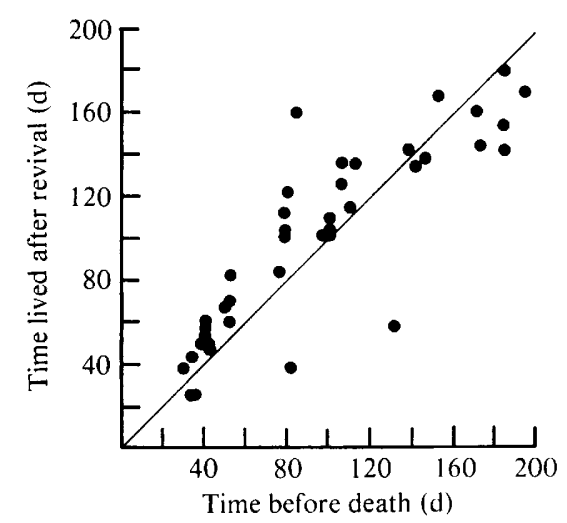

Fig. 1. Scatter plot of the remaining life spans of revived sclerotia of the genotype 2.7 as a function of the time before death of the initial culture used to form each sclerotium. Both revived and initial cultures were grown under normal conditions on rolled oats medium. The line represents the theoretical relationship if the mechanism for the timing of death was suspended during the chronological time as sclerotia. Each point represents a single plasmodial culture.

timing of senescence, and the age of the initial culture before sclerotization did not influence the revived sclerotia from living for a constant total life span (Fig. 1).

If the chronological period as a sclerotium has no effect on vegetative longevity, it would be anticipated that cultures could undergo repeated sclerotium formation while still maintaining a constant total life span. This was tested in multiple crosses of the genotype 2.7 where selected individuals were sclerotized for various intervals. With one exception, sclerotial subdivisions exhibited mean life spans that were similar to the unsclerotized initial group (Fig. 2). Similar results were observed with the genotype 10.76 .

Previously (Lott \& Clark, 1980) we have observed that the creation of normal subdivisions from a single culture results in increased longevity variability among the newly formed group, and that this variability decreases as a function of the age of the initial culture. Selected individuals with life spans greater than the mean can, therefore, be resubdivided and longer-than-average-lived individuals again selected. Although it would be of interest to determine how long a subdivision lineage could be maintained, this type of protocol is impractical in that there is no method for predetermining individual life spans. In order to ensure the creation of a longer-than-average-lived culture, randomly selected individuals must be subdivided and all derived cultures maintained. However, sclerotial subdivision populations offer the advantage that cultures can be kept as sclerotia until the death of the 


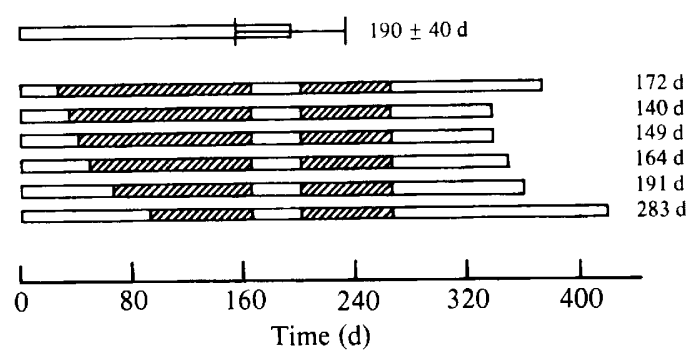

Fig. 2. Total life spans of cultures of the genotype 2.7 as plasmodia (unshaded areas) when sclerotized at various times and kept for different periods as sclerotia (shaded areas). The top bar shows the mean life span ( \pm s.D.) of the initial culture and unsclerotized subdivisions $(n=10)$.

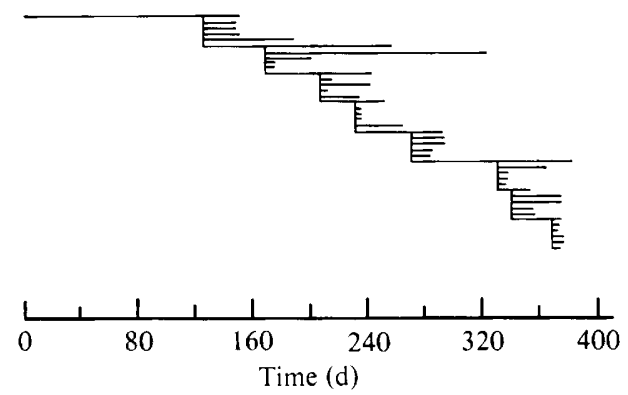

Fig. 3. Total periods of plasmodial existence for cultures of the genotype 62.76 , derived from a single initial individual. Each subdivision population was sclerotized and revived upon the death of the culture used to create the subdivision. Vertical bars represent the times of sclerotization.

initial culture, and an estimation of the mean days of remaining life can be made for the revived population. Cultures living longer than this estimated mean can then be selected for resubdivision. Using this procedure, it was of interest to determine the maximum life span of a sclerotized subdivision lineage, where each subdivision population contained the same number of individuals. To ensure a starting population of short-lived cultures, isolated gametic clone 62 from the sporulated genotype 10.76 was backcrossed to clone 76 , creating the genotype 62.76. Multiple-cross populations were observed to have a mean life span of $72 \pm 19$ (s.D.) d. A longer-than-average-lived single culture from the original population was used to create successive sclerotial subdivision populations (Fig. 3). It was found that the maximum time for continuous plasmodial existence within the subdivision lineage was $375 \mathrm{~d}$. Moreover, it was generally observed that subdivision variability decreased over time.

\section{DISCUSSION}

Ageing in the myxomycetes is unique in that a plasmodium will grow vigorously for extended periods before exhibiting any overt signs of necrosis. Assuming a constant synchronous cell cycle of approximately $12 \mathrm{~h}$ (Lovely \& Threlfall, 1978), it is possible that long-lived genotypes undergo an average of 400 mitotic divisions before death. In most cases the senescent phase is relatively short in comparison with the preceding period of growth. This suggests the presence of a surreptitious mechanism that would, at some point, trigger the onset of necrosis.

The timing mechanism for ageing does not appear to be affected by any cellular alterations involved in sclerotium formation, although many are known to occur during the transition. These include an increase in lipids and sulphonated galactose (McCormick et al., 1970; 
Sullivan, 1953), and differences in non-histone chromosomal proteins (LeStourgeon et al., 1973). Moreover, the chronological time as a sclerotium does not influence the pattern of plasmodial longevity. Considering the observed absence of $\mathrm{O}_{2}$ utilization following dehydration, it can be inferred that metabolism has some function in determination of the life span. Yet short- and long-lived genotypes exhibit little difference in the rate of $\mathrm{O}_{2}$ consumption, suggesting that metabolism does not solely govern the timing of senescence.

A second property of plasmodial ageing is the variation of life spans within a population of isogenic individuals (Lott \& Clark, 1980). It would be expected that if longevity were governed solely by genotype, life spans would be highly similar. However, multiple-cross populations exhibit a linear decline in the ability to survive over time (Lott \& Clark, 1980), suggesting that, in addition, some form of stochastic mechanism operates to determine longevity.

Mean population life spans could, therefore, be centred around a given probability of death. The observation that subdivision populations established from a relatively old initial culture show a decreased variance in remaining life (Lott \& Clark, 1980) could be interpreted as simply the elimination of the probability of young culture deaths.

It appears that the life span variance of sclerotized populations is similar to that of non-sclerotized, subdivision populations. Although selected individuals live longer than the mean, population variances tend to decrease as a function of total culture age. Thus, there seems to be a limit to how long cultures can be maintained in this manner.

It is possible that the mechanism of plasmodial senescence is similar to the observed ageing in the ascomycete Podospora anserina. Ageing in this species is governed, in part, by two nuclear genes, incoloris and vivax (Esser \& Keller, 1976). However, the relationship between the amount of hyphal growth and the onset of necrosis has been interpreted as being stochastic (Smith \& Rubenstein, 1973), and additional work suggests that mitochondria are involved in the ageing process (Tudzynski \& Esser, 1977). We are presently determining how many, and to what extent, nuclear genes govern plasmodial ageing in $D$. iridis.

\section{REFERENCES}

Clark, J. \& HaKim, R. (1980). Ageing of plasmodial heterokaryons in Didymium iridis. Molecular and General Genetics 178, 419-422.

Collins, O. (1961). Heterothallism and homothallism in two myxomycetes. American Journal of Botany 48, 674-683.

DANiel, J. \& Baldwin, H. (1964). In Methods in Cell Physiology 1D, 9-40.

DEE, J. (1975). Slime molds in biological research. Science Progress 62, 523-542.

Esser, K. \& Keller, W. (1976). Genes inhibiting senescence in the ascomycete Podospora anserina. Molecular and General Genetics 144, 107-110.

Jump, J. (1954). Studies of sclerotization in Physarum polycephalum. American Journal of Botany 41, 561-567.

LeStourgeon, W., Nations, C. \& Rusch, H. (1973). Temporal synthesis and intranuclear accumulation of the nuclear acidic proteins during periods of chromatin reactivation in Physarum polycephalum. Archives of Biochemistry and Biophysics 159, 861-872.

LoTT, T. \& ClARK, J. (1980). Plasmodial senescence in the acellular slime mold Didymium iridis. Experimental Cell Research 128, 455-457.

Lovely, J. \& Threlfall, R. (1978). Adenylate cyclase and cyclic AMP phosphodiesterase activity during the mitotic cycle of Physarum polycephalum. Biochemical and Biophysical Research Communications 85, 579-584.

McCormick, J., Blomeuist, J. \& Rusch, H. (1970). Isolation and characterization of an extracellular polysaccharide from Physarum polycephalum. Journal of Bacteriology 104, 1100-1118.

McCullough, C., Cooke, D., Foxon, J., Sudberry, P. \& GRANT, W. (1973). Nuclear DNA content and senescence in Physarum polycephalum. Nature New Biology 245, 263-265.

Poulter, R. (1969). Senescence in Physarum polycephalum. Ph.D. thesis, University of Leicester, U.K.

Smith, J. R. \& Rubenstein, I. (1973). Cytoplasmic inheritance of the timing of 'senescence' in Podospora anserina. Journal of General Microbiology 76, 297-304.

Sullivan, A. (1953). Some aspects of the biochemistry of dormancy in the myxomycete Physarum polycephalum. Physiologia plantarum 6, 804-815.

TUDZYNSKI, P. \& ESSER, K. (1977). Inhibitors of mitochondrial function prevent senescence in the ascomycete Podospora anserina. Molecular and General Genetics 153, 111-113. 